\title{
An emerging recombinant human enterovirus 71 responsible for the 2008 outbreak of Hand Foot and Mouth Disease in Fuyang city of China
}

\author{
Yan Zhangt1, Zhen Zhu+1, Weizhong Yang ${ }^{+2}$, Jun Ren+3, Xiaojuan Tan+1, Yu Wang2, Naiying Mao', Songtao Xu',

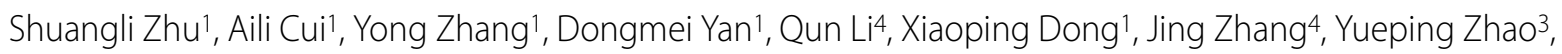 \\ Junfeng Wan ${ }^{5}$, Zijian Feng ${ }^{4}$, Junling Sun ${ }^{4}$, Shiwen Wang ${ }^{1}$, Dexin Li*1 and Wenbo Xü ${ }^{* 1,2}$
}

\begin{abstract}
Hand, foot and mouth disease (HFMD), a common contagious disease that usually affects children, is normally mild but can have life-threatening manifestations. It can be caused by enteroviruses, particularly Coxsackieviruses and human enterovirus 71 (HEV71) with highly variable clinical manifestations. In the spring of 2008, a large, unprecedented HFMD outbreak in Fuyang city of Anhui province in the central part of southeastern China resulted in a high aggregation of fatal cases. In this study, epidemiologic and clinical investigations, laboratory testing, and genetic analyses were performed to identify the causal pathogen of the outbreak. Of the 6,049 cases reported between 1 March and 9 May of 2008, 3023 (50\%) were hospitalized, 353 (5.8\%) were severe and 22 (0.36\%) were fatal. HEV71 was confirmed as the etiological pathogen of the outbreak. Phylogenetic analyses of entire VP1 capsid protein sequence of 45 Fuyang HEV71 isolates showed that they belong to C4a cluster of the C4 subgenotype. In addition, genetic recombinations were found in the $3 \mathrm{D}$ region (RNA-dependent RNA polymerase, a major component of the viral replication complex of the genome) between the Fuyang HEV71 strain and Coxsackievirus A16 (CV-A16), resulting in a recombination virus. In conclusion, an emerging recombinant HEV71 was responsible for the HFMD outbreak in Fuyang City of China, 2008.
\end{abstract}

\section{Background}

The first HEV71 infection was reported in 1969 in the US [1]. Although HFMD is usually a mild disease, several HEV71 outbreaks associated with severe neurological complications have been reported in Western Pacific countries or regions, including Malaysia in 1997; Taiwan in 1998, 2000 and 2001; Australia in 1999; Singapore in 2000; Japan in 1997 and 2000; and Shandong of China in 2007 [2-8].

Human enterovirus 71 (HEV71) is one of the member in HEV-A species of Enterovius genus in the family Picornaviridae. The genome of HEV71 consists of a single-

\footnotetext{
*Correspondence: lidx@chinacdc.cn, wenbo_xu1@yahoo.com.cn

${ }^{1}$ Key Laboratory for Molecular Virology \& Genetic Engineering, National Institute for Viral Disease Control and Prevention, Chinese Center for Disease Control and Prevention, Beijing 100052, China

${ }^{2}$ Chinese Center for Disease Control and Prevention, Beijing 100050, China + Contributed equally

Full list of author information is available at the end of the article
}

stranded positive-sense RNA of approximately 7400 nucleotides. The viral genome contains a $5^{\prime}$ - and $3^{\prime}-$ untranslated regions (UTRs) which are essential for viral RNA replication. The genome is translated as a single large polyprotein that is composed of four capsid proteins, VP1 to VP4, and seven nonstructural proteins, 2A, 2B, 2C, 3A, 3B, 3C, and 3D. VP1 to VP4 capsid proteins were encoded by $\mathrm{P} 1$ region. The $\mathrm{P} 2$ and $\mathrm{P} 3$ regions encode nonstructural proteins and the RNA-dependent RNA polymerase, $3 \mathrm{D}$, is a major component of the viral replication complex [9].

Sporadic epidemic or outbreaks of HEV71 infection had occurred in mainland of China since 1995[8,10,11], but the highly aggregated cases of rapidly fatal pediatric infections occurred in the Fuyang HFMD outbreak in 2008 is unprecedented. The identification of the causal pathogen is crucial for preventing disease spread, reducing fatality rate, and better understanding the pathogenic 
mechanism underlying disease severity. This study reported the investigation and the etiological identification of the Fuyang HFMD outbreak in 2008. And the genetic recombination event was also found between the Fuyang HEV71 isolates and CV-A16.

\section{Results}

\section{Cases and epidemiology}

This study focused on the outbreak between 1 March and 9 May, 2008. A total of 6,049 HFMD cases were reported from local healthcare facility in Fuyang city (Figure 1), of which 3,023 (50\%) were hospitalized, $353(6 \%)$ were severe and $22(0.4 \%)$ were fatal. The incidence rate was $70 / 100,000$; while the fatality rate was $0.4 \%$. The gender ratio of the epidemics was $1.9: 1$, with 3,938 male and 2,111 female cases. The age ranged from 28 days to 18 years, with $78 \%$ of the cases $<3$ years old. All of the 22 fatal cases were $<3$ years old, and the youngest was 3 months.

\section{Case definitions}

The mild HFMD outpatients were defined as patients with fever and vesicular lesions on their palms, feet and mouth. Severe cases usually presented with neurologic complications, which were defined as having two of fol- lowing clinical manifestations: brainstem encephalitis or aseptic meningitis; continuous high fever (temperature of at least $38^{\circ} \mathrm{C}$ ); weakness, vomiting, irritability, myoclonus and acute flaccid paralysis; pulmonary edema or hemorrhage, heart and lung failure. Encephalitis, aseptic meningitis, pulmonary edema, pulmonary hemorrhage, acute flaccid paralysis, myocarditis were characterized by the same definition as described previously [2].

\section{Clinical description}

General clinical symptoms of the Fuyang HFMD outbreak included rash, fever, general malaise, cough, vomiting and neurologic complications (such as encephalitis, aseptic meningitis, or acute flaccid paralysis). The analysis of the clinical characteristics of 15 out of 22 fatal cases showed that these cases had an acute onset of fever, sore throat, and myalgia (influenza-like illness) without catarrhal syndrome, but the rash was rare. The conditions of most of cases subsequently deteriorated, in which the patients developed tachypnea, cyanosis, and some presented seizures with white or pink foaming at the mouth (Table 1). Most hospitalized cases were initially diagnosed as severe pneumonia, some patients died within 15 days after onset.

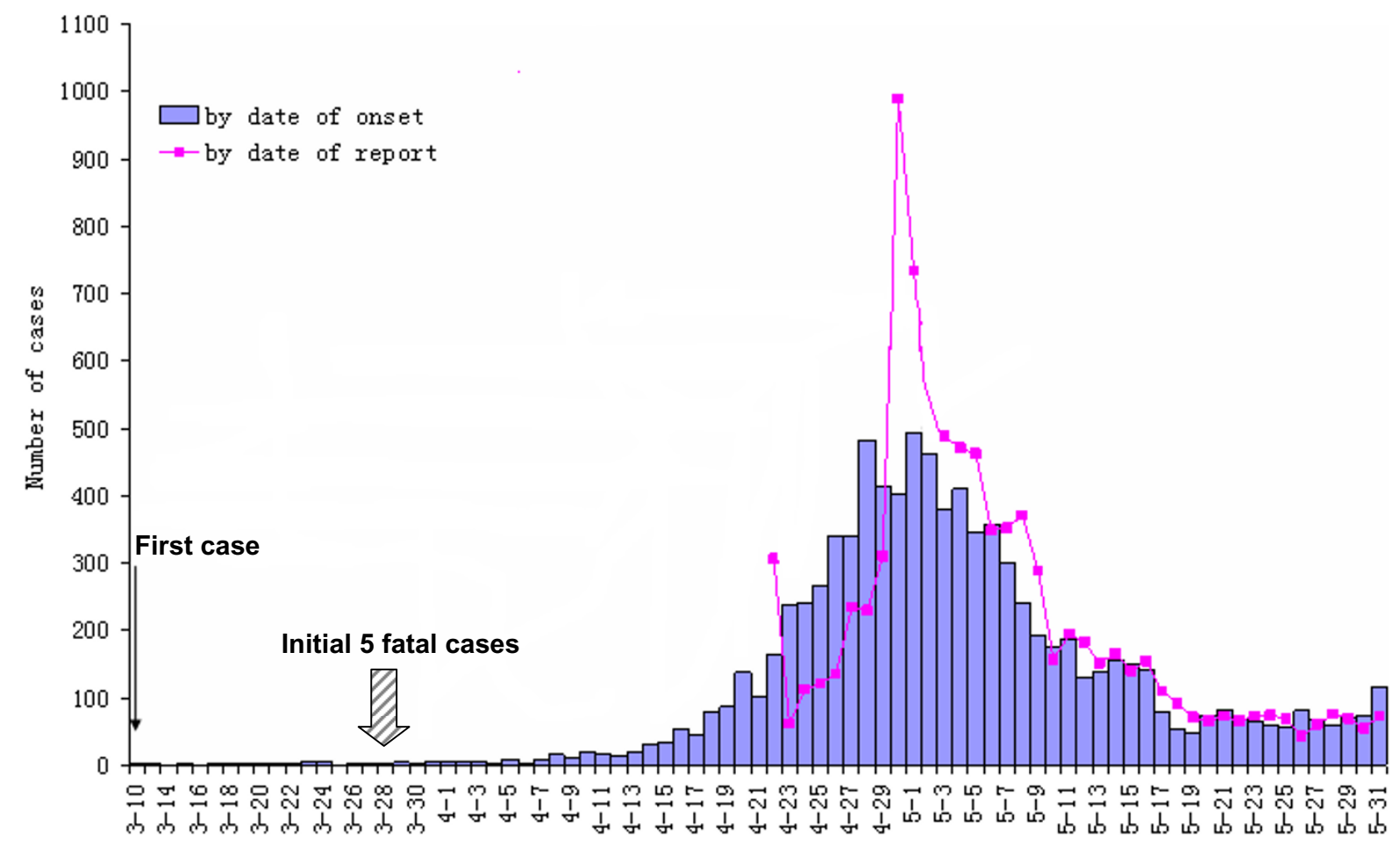

Figure 1 Distribution of hand, foot, and mouth disease (HFMD) patients in Fuyang City, Anhui province, by date. By 31 May 2008 , the outbreak had affected 6883 children (This study focused on the outbreak between March 1 and May 9 of 2008, which included 6049 reported cases). The bold slipped arrows indicated the death dates of initial 5 fatal cases. 
Table 1: Clinical symptoms of fatal HFMD cases in Fuyang hospital $(n=15)$.

\begin{tabular}{lcc}
\hline \multicolumn{1}{c}{ Clinical symptom } & Number of cases & Proportion (\%) \\
\hline Fever & 15 & 100 \\
Tachypnea & 14 & 93.3 \\
Oral cyanosis & 12 & 80 \\
Pink foaming at the mouth & 9 & 60 \\
Coughing & 7 & 46.7 \\
Vomiting & 8 & 53.3 \\
Myoclonic twitching & 5 & 33.3 \\
Tiny pink rash on palm, sole & 2 & 13.3 \\
Nasal discharge & 2 & 13.3 \\
Stiff neck & 2 & 13.3 \\
\hline
\end{tabular}

\section{Laboratory identification of the etiologic pathogen of the outbreak}

Since most of fatal or severe cases were characterized as respiratory disease syndrome, direct RT-PCR were performed on a total of 89 specimens (including blood sera, throat swabs and autopsy tissue samples) collected from fatal and severe cases to detect for seasonal influenza, avian influenza A/H5N1, Severe Acute Respiratory Syndrome (SARS), RSV, Adeno virus, and other respiratory bacteria microorganism, and all the results were negative. As enterovirus infection could also induce the respiratory symptoms, the specimens were also tested using primers for pan-enterovirus, HEV71, CV-A16 and Echovirus. Positive results were obtained only for pan-enterovirus and HEV71. Further investigation using sequence determination and BLAST sequence analysis revealed that all of the sequences had high homology (95.1\%) with HEV71 (strain E2004104-TW-CDC, accession number EF373576). To further confirm the possible etiological pathogen, additional 121 more clinical specimens were collected for the detection of HEV71 and CV-A16 using direct RT-PCR.

Thus, a total of 210 specimens collected from 13 fatal cases, 99 severe cases and 39 mild cases, respectively, had been tested for HEV71 and CV-A16 using direct RTPCR. Six (46\%) of 13 fatal cases, 36 (36\%) of 99 severe cases, 17 (44\%) of 39 mild cases were positive for HEV71 (Table 2). None were positive for CV-A16 infection. Gene sequencing studies of HEV71 showed that sequences from fatal, severe and mild cases exhibited high homology.

All of the 210 specimens were separately cultured in RD and HEp- 2 cell lines. A total of 50 enterovirus isolates were obtained from clinical specimens: 34 of $132(26 \%)$ throat swabs, 7 of 12 (58\%) stool samples, 3 of 4 (75\%) vesicular fluid, 3 of 22 (14\%) anus swab, 1 of 2 (50\%) antemortem oral efflux, and 2 of 23 (9\%) autopsy lung tissue.
No virus was isolated from CSF and other autopsy tissue samples. By RT-PCR and sequence determination, 45/50 (90\%) of the enteroviruses were identified as HEV71. In addition, 2 Echovirus 9 (E9), 1 Coxsackievirus A4 (CVA4), 1 Coxackievirus A9 (CV-A9) and 1 Coxackievirus B3 (CV-B3) were isolated and identified. No CV-A16 virus was found.

Taken together, the results showed that HEV71 was associated with the Fuyang HFMD outbreak in 2008.

\section{Phylogenetic analysis of entire VP1 region of HEV71}

The entire VP1 sequences of 45 HEV71 isolates from 6 fatal cases, 26 severe cases and 13 mild outpatients were used for phylogenetic analysis(data now shown). The nucleotide and the amino acid homologies among the Fuyang isolates were $96.4 \%-100 \%$ and $98.7 \%-100 \%$, respectively. The average nucleotide homologies within fatal, severe and mild cases were $99.3 \%, 99.7 \%$ and $98.9 \%$, respectively. Based on the VP1 gene, the nucleotide and amino acid homology between fatal or severe cases and mild cases were $96.4 \%-100 \%$ and $98.7 \%-100 \%$, respectively. No significant nucleotide and amino acid differences in the VP1 region were found between severe or fatal cases and mild cases.

To determine the molecular epidemiology of the Fuyang HEV71 isolates, a phylogenetic dendrogram was constructed with 14 representative Fuyang HEV71 isolates (which were selected based on the case classification and their nucleotide divergence: 4 fatal cases, 8 severe cases, 2 mild cases), 43 HEV71 strains available in the GenBank isolated from 12 provinces in mainland of China between 1997-2008, and 18 international HEV71 strains that represented all 11 known genotypes or subgenotypes (A, B1B5, C1-C5), and 2 HEV71 strains from 2 fatal French cases of 2007-2008 (Table 3, Figure. 2). It showed that the Fuyang HEV71 isolates belonged to cluster C4a of the subgenotype C4, which was similar to the HEV71 
Table 2: Case classification and HEV71 positive number (rate) by RT-PCR or virus isolation.

\begin{tabular}{|c|c|c|c|c|c|}
\hline \multirow[b]{2}{*}{$\begin{array}{c}\text { Case } \\
\text { Classification }\end{array}$} & \multirow[b]{2}{*}{ No. of Cases } & \multicolumn{2}{|c|}{ HEV71 } & \multicolumn{2}{|c|}{ Other Enterovirus } \\
\hline & & direct RT-PCR & virus Isolation & $\begin{array}{c}\text { direct RT-PCR for } \\
\text { CV-A16 }\end{array}$ & virus Isolation \\
\hline Fatal cases & 13 & $6(46 \%)$ & $6(46 \%)$ & 0 & 0 \\
\hline Severe cases & 99 & $36(36 \%)$ & $26(26 \%)$ & 0 & 1 (E9), 1(CV-A4) \\
\hline Mild outpatients & 39 & $17(44 \%)$ & $13(33 \%)$ & 0 & $\begin{array}{c}1 \text { (E9), } 1(\mathrm{CV}-\mathrm{B} 3), \\
1(\mathrm{CV}-\mathrm{A} 9)\end{array}$ \\
\hline Total & 151 & $59(39 \%)$ & $45(30 \%)$ & 0 & 5 \\
\hline
\end{tabular}

Note: E9: Echovirus 9; CV-A4: Coxackievirus A4; CV-B3: Coxackievirus B3; CV-A9:Coxackievirus A9. All the positive PCR fragments and isolates were confirmed by sequencing.

sequences isolated from the mainland of China during 2003-2008 (including the sequences from Shandong Province in 2007 Linyi HFMD outbreak(Zhang, 2009 \#233)).

The average nucleotide homology between Fuyang isolates in this study and those isolated from other provinces in China in 2008, and between Fuyang isolates in this study and the representative sequences from Shandong Province in 2007 Linyi HFMD outbreak were $98.5 \%$ and $97.1 \%$, respectively.

\section{Identification of recombination between HEV71 and CV- A16}

The phylogenetic analysis for $3 \mathrm{D}$ region revealed that the sequences of representative isolates from 4 fatal cases, 3 severe cases and 1 mild case shared $77.7 \%-78.1 \%$ and 92.0\%-92.2\% identities in nucleotides and amino acids, respectively, with the $\mathrm{HEV} 71$ prototype strain $\mathrm{BrCr}$; while they shared $84.6-85.0 \%$ and $97.4-97.6 \%$ identities in nucleotides and amino acids, respectively, with the CVA16 prototype strain G-10. The phylogenetic tree of the $3 \mathrm{D}$ gene showed that the 8 sequences were clustered into the same group and were closer to strain G-10 than to strain $\mathrm{BrCr}$ (Figure 3A).

In order to identify the possible recombination events, one of the Fuyang representative strain of HEV71: EV71/ Fuyang.Anhui.CHN/17.08/1 was sequenced for the entire genome, and similarity plot and bootscan analysis were used to study the relationship of the Fuyang representative strain of HEV71 to the prototype strains of CV-A16 and HEV71, and genotype $B$ representative strain of HEV71 (Figure 3B). In the analysis, the sequence of the entire genome of the Fuyang strain was used as the query sequence and compared to the sequences of the CV-A16 prototype strain $\mathrm{G}-10$, the $\mathrm{HEV} 71$ prototype strain $\mathrm{BrCr}$ and $B$ genotype representative strain: MS/7423/87. The results showed that the $\mathrm{P} 1$ region of the Fuyang strain had the highest degree of similarity to the sequence of $\mathrm{BrCr}$, and its $\mathrm{P} 2$ region and $3 \mathrm{~A}, 3 \mathrm{~B}, 3 \mathrm{C}$ region exhibited the highest degree of similarity to the sequence of G-10 and MS/7423/87, while 3D region showed the highest degree of similarity to the sequence of G-10, which suggested that the Fuyang strain might be a recombinant virus with CV-A16 in 3D region.

\section{Discussion}

The diagnosis of virological etiology is critical and challenging at the beginning of the HFMD outbreak. During the Fuyang 2008 outbreak, because the clinical symptoms of HFMD showed atypical manifestations, which was different from common cutaneous lesions, local doctors misdiagnosed HFMD as pneumonia. In addition, the identification of HEV71 infection was hampered by the sudden death of 22 children within 5 days of pulmonary edema or hemorrhage, heart and lung failure. RT-PCR and sequencing were primarily performed to identify the virological etiology of the outbreak. Laboratory testing showed that $39 \%$ of the cases were confirmed as HEV71 infection. Together with clinical manifestations and epidemiological data, we concluded that HEV71 was identified as the major etiological pathogen of the Fuyang HFMD outbreak.

In the Fuyang HFMD outbreak, diseases were manifested with cutaneous lesions in mild cases and CNS symptoms in fatal or severe cases. The findings were consistent with studies of the 1997 outbreak in Malaysia and the 1998 outbreak in Taiwan, in which manifestations of HEV71 infections predominate with neurological complications in fatal or severe cases[2,3]. Analyses of the VP1 sequence showed high homology between severe or fatal cases and mild HFMD cases and no significant nucleotide differences were found. Other groups have also studied the complete genome of HEV71 isolated from fatal and non-fatal HFMD cases and found no genetic differences that could be correlated with the severity of clinical manifestation[12,13]. Studies on the pathogenic mechanism for HEV71 have pointed to altered innate immunity. An 

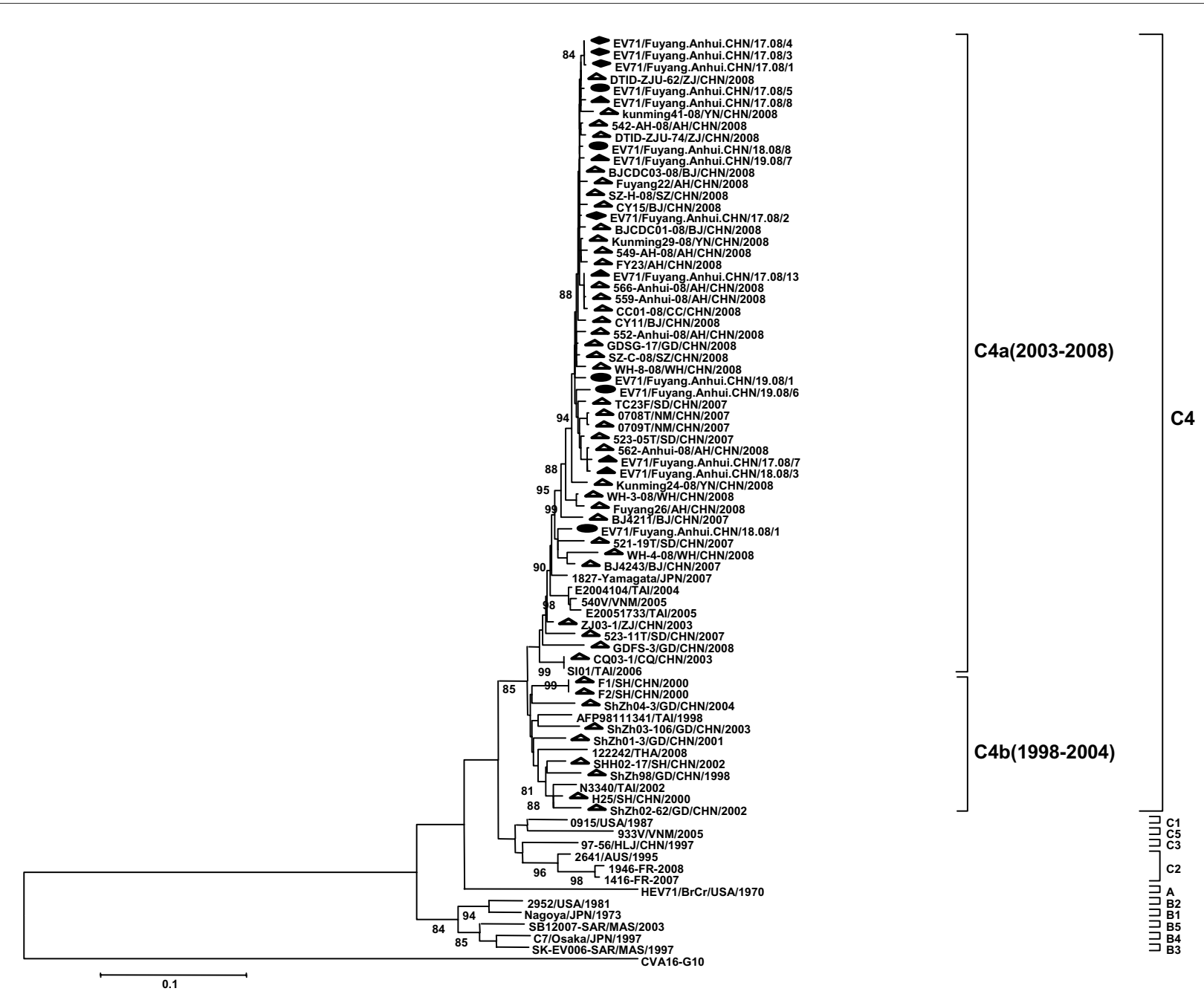

Figure 2 Phylogenetic dendrogram were drawn on the basis of the 891-nt sequence of entire VP1 gene using the Neighbor-Joining method of MEGA software (version 4.0) for 14 selected Fuyang representative isolates and other HEV71 strains of known subgenotypes listed in Table 1. The bootstrap values from 1000 pseudoreplicates for major lineages within the tree are shown as percentages. The prototype CVA16 strain (G-10) was used as outgroup. Solid diamond indicated the fatal cases; Solid triangle indicated the severe cases; Solid round indicated the outpatients; Triangle indicated the HEV71 sequences from other provinces in China between 1997-2008 available in GenBank database.

epidemiologic study during the same outbreak implicated treatment of initial fever of HEV71 infections with glucocorticoids, which block the innate immune response, as contributing to the high rate of severe disease[14]. Experimental animal and clinical studies also support this finding indicating that weakened innate immunity is associated with severe disease and death from HEV71 infection[15-17]. This in combination with introduction of the new recombinant virus may help to explain the increased severity of the Fuyang outbreak.

In this study, genetic recombination event were found between the Fuyang HEV71 isolates, including both fatal cases and non-fatal cases, and CV-A16, which was similar to other studies[18,19]. For enteroviruses[20] and dengue viruses[21], genetic recombination is a well-known phe- nomenon and recombination could result in the emergence of viruses with altered pathogenic potentials. Except for the Fuyang HEV71 isolates, genetic recombination was also found in the HEV71 viruses, circulated in mainland of China during 1998-2008. However, it was very difficult to clarify the time of recombination and the role of the HEV71 recombinant in HFMD outbreaks remains unclear.

Previous studies showed that all known HEV71 strains could be divided into three distinct genogroups $(\mathrm{A}, \mathrm{B}, \mathrm{C})$ and 10 subgenogroups (A, B1-5, C1-4) based on VP1 gene sequences; the subgenotype $\mathrm{C} 4$ could be further divided into $\mathrm{C} 4 \mathrm{a}$ and $\mathrm{C} 4 \mathrm{~b}$ clusters[8]. Based on the phylogenetic analyses, Fuyang isolates belonged to $\mathrm{C} 4 \mathrm{a}$ cluster of the subgenotype $\mathrm{C} 4$ and showed high homology with 
Table 3: Entire VP1 gene nucleotide sequences of the HEV71 strains used to generate the HEV71 phylogenetic dendrograms.

\begin{tabular}{|c|c|c|c|c|c|}
\hline Strain & Source & GenBank No. & Strain & Source & GenBank No. \\
\hline HEV71/BrCr/USA/70 & GenBank & $\underline{\text { U22521 }}$ & $\mathrm{TC} 23 \mathrm{~F} / \mathrm{SD} / \mathrm{CHN} / 2007$ & GenBank & EU753417 \\
\hline CVA16/G-10 & GenBank & $\underline{\mathrm{U} 05876}$ & $\mathrm{CY} 11 / \mathrm{BJ} / \mathrm{CHN} / 2008$ & GenBank & FJ469153 \\
\hline Nagoya/JPN/73 & GenBank & $\underline{A B 059813}$ & $\mathrm{CY} 15 / \mathrm{BJ} / \mathrm{CHN} / 2008$ & GenBank & FJ469154 \\
\hline 2952/USA/81 & GenBank & $\underline{\text { AF135888 }}$ & DTID-ZJU-62/ZJ/CHN/2008 & GenBank & $\underline{F J 158600}$ \\
\hline 0915/USA/87 & GenBank & $\underline{\text { AF009549 }}$ & DTID-ZJU-74/ZJ/CHN/2008 & GenBank & $\underline{F J 158600}$ \\
\hline 2641/AUS/95 & GenBank & AF135947 & GDFS-3/GD/CHN/2008 & GenBank & FJ194964 \\
\hline SK-EV006-SAR/MAS/97 & GenBank & $\underline{\mathrm{AB} 059819}$ & GDSG-17/GD/CHN/2008 & GenBank & $\underline{\text { FJ194965 }}$ \\
\hline C7/Osaka/JPN/97 & GenBank & AB059818 & $\mathrm{FY} 23 / \mathrm{AH} / \mathrm{CHN} / 2008$ & GenBank & EU812515 \\
\hline SB12007-SAR/MAS/03 & GenBank & AY905548 & Fuyang22/AH/CHN/2008 & GenBank & EU913466 \\
\hline 933V/VNM/05 & GenBank & $\underline{\mathrm{AM} 49061}$ & Fuyang26/AH/CHN/2008 & GenBank & $\underline{E U 913468}$ \\
\hline 540V/VNM/05 & GenBank & $\underline{\text { AM490151 }}$ & WH-4-08/WH/CHN/2008 & GenBank & FJ765433 \\
\hline SI01/THA/06 & GenBank & $\underline{E F 203407}$ & WH-3-08/WH/CHN/2008 & GenBank & FJ765432 \\
\hline 1827-Yamagata/JPN/07 & GenBank & $\underline{A B 433890}$ & Kunming24-08/YN/CHN/2008 & GenBank & FJ765425 \\
\hline 122242/THA/08 & GenBank & FJ151494 & $562 \mathrm{AH}-08 / \mathrm{AH} / \mathrm{CHN} / 2008$ & GenBank & FJ765420 \\
\hline AFP98111341/TAI/98 & GenBank & $\underline{\mathrm{DQ} 841953}$ & WH-8-08/WH/CHN/2008 & GenBank & $\underline{F J 765434}$ \\
\hline N3340/TAl/98 & GenBank & $\underline{E U 131776}$ & SZ-C-08/SZ/CHN/2008 & GenBank & FJ765429 \\
\hline E2004104/TAI/04 & GenBank & DQ841964 & 552-AH-08/AH/CHN/2008 & GenBank & $\underline{F J 765418}$ \\
\hline E20051733/TAl/05 & GenBank & $\underline{\mathrm{DQ} 841971}$ & $\mathrm{CC} 01-08 / \mathrm{CC} / \mathrm{CHN} / 2008$ & GenBank & $\underline{F J 765424}$ \\
\hline 1946-FR-2008 & GenBank & $\underline{\text { FJ824736 }}$ & 559-AH-08/AH/CHN/2008 & GenBank & $\underline{F J 765419}$ \\
\hline 1416-FR-2007 & GenBank & $\underline{F J 824734}$ & 549-AH-08/AH/CHN/2008 & GenBank & $\underline{\text { FJ765417 }}$ \\
\hline 97-56/HLJ/CHN/97 & GenBank & AB115494 & Kunming $29-08 / \mathrm{YN} / \mathrm{CHN} / 2008$ & GenBank & FJ765426 \\
\hline ShZh98/GD/CHN/98 & GenBank & $\underline{\text { AF302996 }}$ & $\mathrm{BJCDC01-08/BJ/CHN/2008}$ & GenBank & $\underline{\text { FJ765424 }}$ \\
\hline $\mathrm{H} 25 / \mathrm{SH} / \mathrm{CHN} / 2000$ & GenBank & $\underline{A B 115492}$ & $\mathrm{SZ}-\mathrm{H}-08 / \mathrm{SZ} / \mathrm{CHN} / 2008$ & GenBank & $\underline{\text { FJ765430 }}$ \\
\hline $\mathrm{F} 1 / \mathrm{SH} / \mathrm{CHN} / 2000$ & GenBank & $\underline{A B 115490}$ & 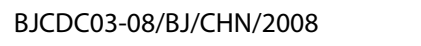 & GenBank & $\underline{F J 765423}$ \\
\hline $\mathrm{F} 2 / \mathrm{SH} / \mathrm{CHN} / 2000$ & GenBank & $\underline{A B 115491}$ & EV71/Fuyang.Anhui.CHN/17.08/1 & $\begin{array}{l}\text { This } \\
\text { study }\end{array}$ & EU703812 \\
\hline ShZh01-3/CHN/2001 & GenBank & $\underline{\text { AY } 895132}$ & EV71/Fuyang.Anhui.CHN/17.08/2 & $\begin{array}{l}\text { This } \\
\text { study }\end{array}$ & EU703813 \\
\hline $\mathrm{SHH} 02-17 / \mathrm{SH} / \mathrm{CHN} / 2002$ & GenBank & $\underline{\text { AY5 } 547500}$ & EV71/Fuyang.Anhui.CHN/17.08/3 & $\begin{array}{l}\text { This } \\
\text { study }\end{array}$ & EU703814 \\
\hline ShZh02-62/CHN/2002 & GenBank & $\underline{\text { AY } 895136}$ & EV71/Fuyang.Anhui.CHN/17.08/5 & $\begin{array}{l}\text { This } \\
\text { study }\end{array}$ & $\underline{\mathrm{GQ} 121418}$ \\
\hline ZJ03-1/ZJ/CHN/2003 & GenBank & $\underline{\text { AY } 905614}$ & EV71/Fuyang.Anhui.CHN/17.08/7 & $\begin{array}{l}\text { This } \\
\text { study }\end{array}$ & $\underline{\mathrm{GQ} 121420}$ \\
\hline CQ03-1/CQ/CHN/2003 & GenBank & $\underline{\text { AY5 } 547501}$ & EV71/Fuyang.Anhui.CHN/17.08/8 & $\begin{array}{l}\text { This } \\
\text { study }\end{array}$ & $\underline{G Q 121421}$ \\
\hline $\begin{array}{l}\text { ShZh03-106/GD/CHN/ } \\
2003\end{array}$ & GenBank & $\underline{\mathrm{AY} 895138}$ & EV71/Fuyang.Anhui.CHN/17.08/4 & $\begin{array}{l}\text { This } \\
\text { study }\end{array}$ & $\underline{\mathrm{GQ} 121423}$ \\
\hline ShZh04-3/GD/CHN/2004 & GenBank & $\underline{\text { AY } 895142}$ & EV71/Fuyang.Anhui.CHN/19.08/7 & $\begin{array}{l}\text { This } \\
\text { study }\end{array}$ & $\underline{\text { GQ121424 }}$ \\
\hline 0708T/NM/CHN/2007 & GenBank & EU910861 & EV71/Fuyang.Anhui.CHN/18.08/1 & $\begin{array}{l}\text { This } \\
\text { study }\end{array}$ & $\underline{\mathrm{GQ} 121427}$ \\
\hline 0709T/NM/CHN/2007 & GenBank & EU910862 & EV71/Fuyang.Anhui.CHN/17.08/13 & $\begin{array}{l}\text { This } \\
\text { study }\end{array}$ & $\underline{\mathrm{GQ} 121428}$ \\
\hline
\end{tabular}


Table 3: Entire VP1 gene nucleotide sequences of the HEV71 strains used to generate the HEV71 phylogenetic dendrograms. (Continued)

\begin{tabular}{|c|c|c|c|c|c|}
\hline $\mathrm{BJ} 4211 / \mathrm{BJ} / \mathrm{CHN} / 2007$ & GenBank & EU024958 & EV71/Fuyang.Anhui.CHN/18.08/3 & $\begin{array}{l}\text { This } \\
\text { study }\end{array}$ & GQ121431 \\
\hline $\mathrm{BJ} 4243 / \mathrm{BJ} / \mathrm{CHN} / 2007$ & GenBank & EU019910 & EV71/Fuyang.Anhui.CHN/19.08/1 & $\begin{array}{l}\text { This } \\
\text { study }\end{array}$ & GQ121433 \\
\hline 521-19T/SD/CHN/2007 & GenBank & EU753376 & EV71/Fuyang.Anhui.CHN/18.08/8 & $\begin{array}{l}\text { This } \\
\text { study }\end{array}$ & $\underline{\text { GQ121436 }}$ \\
\hline $523-05 T / S D / C H N / 2007$ & GenBank & EU753397 & EV71/Fuyang.Anhui.CHN/19.08/6 & $\begin{array}{l}\text { This } \\
\text { study }\end{array}$ & $\underline{\text { GQ121441 }}$ \\
\hline $523-11 \mathrm{~T} / \mathrm{SD} / \mathrm{CHN} / 2007$ & GenBank & EU753402 & & & \\
\hline
\end{tabular}

the isolates circulating in other provinces of the mainland of China between 2007 and 2008, including those from the HFMD outbreak in Linyi city in Shandong province in 2007[8]. The C4 subgenotype of HEV71 was initially identified in Guangdong province in 1998 and has been continuously circulating for at least 10 years[10], which may reflect the pattern of endemic circulation of subgenotype $\mathrm{C} 4$ viruses. Interestingly, $\mathrm{C} 4 \mathrm{~b}$ viruses were the predominant circulating strain in mainland of China prior to 2004. Since then, however, C4a viruses have become the predominant strains, which have been continuously circulating and causing epidemic in the mainland of China so far [8].

The subgenotype C4 HEV71 also circulated in neighboring countries and regions, such as, in chronological order, Taiwan, Japan, Vietnam and Thailand[8]; other subgenogroups including B2, B4, B5, C1, C2 co-circulated with C4 between 1990 and 2008[5,7,22]. Interestingly, the subgenotype C4 was the only subgenotype found in China since 1998.

A total of 489,073 HFMD cases, including 126 fatal cases, were reported in China in 2008 (data source: NNDRS). Recombinant HEV71 infection may become more serious public health threat for children under the age of 5 in China, because little is known about the genetics and transmission trend of this fasting mutating virus. To tackle the increasing threat, there is an urgent need for establishing an effective HEV71 surveillance system in China and isolating more viruses, so a complete genetic baseline could be set up for the entire country.

\section{Conclusion}

This study reported the identification of the etiological pathogen of the HFMD ourtbreak in Fuyang city of China, 2008. HEV71 was confirmed to associate with the outbreak. Phylogenetic analyses of entire VP1 capsid protein sequence of 45 Fuyang HEV71 isolates showed that they belong to $\mathrm{C} 4 \mathrm{a}$ cluster of the $\mathrm{C} 4$ subgenotype. In addition, genetic recombinations were found in the 3D region between the Fuyang HEV71 strain and Coxsackie- virus A16 (CV-A16), resulting in a recombination virus. In conclusion, an emerging recombinant HEV71 was responsible for the HFMD outbreak in Fuyang City of China, 2008.

\section{Methods}

\section{Outbreak investigation}

Fuyang city, in the northwest of Anhui Province of China, has 9.76 million inhabitants and a high population density $(1,000$ per square $\mathrm{km})$. All the cases were reported from local healthcare facility of Fuyang city everyday to National Notifiable Disease Report System (NNDRS) of Chinese Center for Disease Control and Prevention (CCDC). And the number of cases and the incidence rates were taken directly from the reports in the NNDRS in China.

\section{Clinical specimens and identification}

Based on the clinical presentations and case definition, the clinical specimens were collected from fatal cases, severe cases and mild cases individually. From March 1 to May 9, 49 specimens were available from 13 fatal cases, including antemortem specimens (throat swabs, serum, oral efflux), autopsy tissues, lung aspirates, CSF; 122 specimens were collected from 99 severe cases, including throat swabs, stool, and vesicular fluid; 39 throat swabs were from 39 mild outpatients. All patients (or the guardians of the children patients or relatives of fatal or comatose patients) gave oral informed consent. Direct reverse transcription-polymerase chain reaction (RT-PCR) was performed using different sets of primers for panenteroviruses[23]; HEV71[8] and CV-A16[8]. Human rhabdomyosarcoma (RD) and human laryngeal carcinoma (HEp-2) cell lines were used to isolate viruses from clinical specimens. Cultures that exhibited a characteristic enterovirus cytopathic effect were evaluated by RT-PCR and sequencing.

\section{Sequence determination of the entire VP1 and 3D gene}

The entire VP1 and 3D gene of the HEV71 isolates from this outbreak were amplified by RT-PCR with in-house 


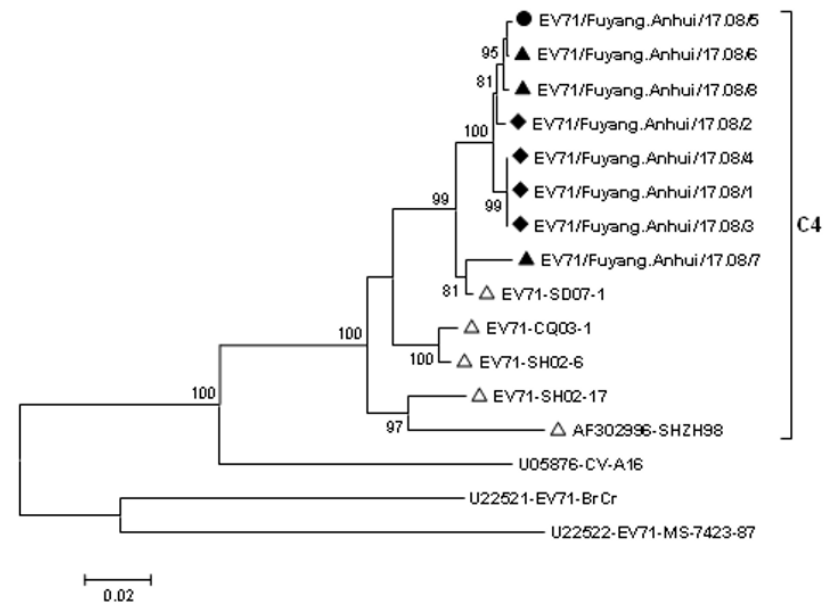

A: Phylogenetic tree for the entire 3D gene
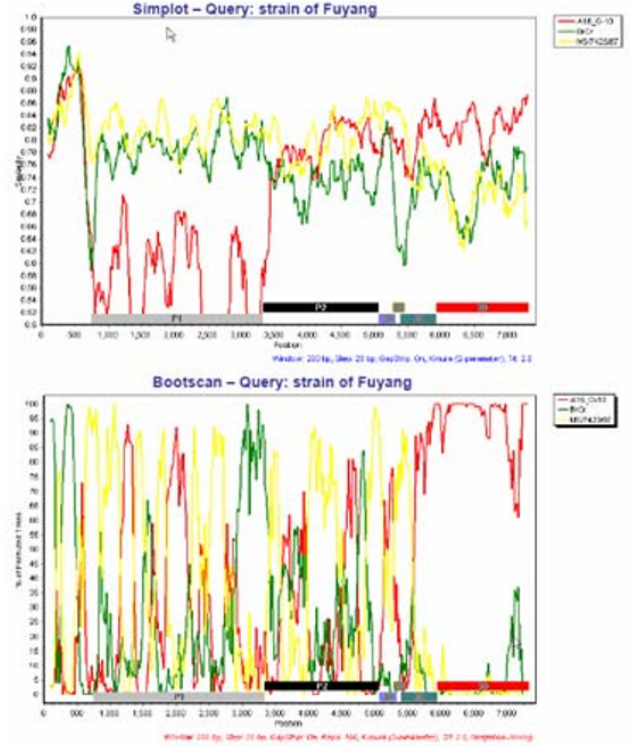

B: similarity plot and bootscan analysis in the genome of HEV71

Figure 3 A: Phylogenetic analysis based on the entire 3D gene. The trees were drawn on the basis of the 1368-nt sequence including the noncoding region using the Neighbor-Joining method of MEGA software (version 4.0). Solid diamond indicated the fatal cases; Solid triangle indicated the severe cases; Solid round indicated the mild cases; Triangle indicated the HEV71 sequences from other provinces in China between 1997 and 2008(available from GenBank). The scale bar indicates the number of nucleotide substitutions per site. B: similarity plot and bootscan identified the recombinant sequences in 3D region of genome of HEV71. The window size of 200-nt slides in increments of $20 \mathrm{nt}$ at a time. The Kimura model with the Jukes-Cantor correction was used. The vertical axis indicates the percent nucleotide identities between strain Fuyang 2008, CV-A16, and HEV71 strain. The horizontal axis indicates the nucleotide positions of the entire genome.

primers that flanked the VP1 gene[8] and 3D gene [HEV71-23S: 5'-ATCACCAAGTTCATACCAGA-3' (nucleotides 5653-5672, relative to strain $\mathrm{HEV} 71 / \mathrm{BrCr}$ ), and HEV71-28A: 5'-GCTATTCTGGTTATAACAAA-3' (nucleotides 7403-7422, relative to strain $\mathrm{HEV71/BrCr}$ )]. The RT-PCR reactions were performed using an Access RT-PCR Kit (Promega, USA). The PCR products were purified using a QIAquick Gel Extraction Kit (Qiagen), and the amplicons were directly sequenced from double direction using an ABI PRISM 3100 Genetic Analyzer (Applied Biosystems, Hitachi, Japan).

\section{Nucleotide sequence accession numbers}

The entire VP1 sequences of 28 representative Fuyang isolates in this study were deposited in the GenBank database under the accession numbers: EU703812-EU703814,
GQ121417-GQ121441 (Table 1). And 3D nucleotide sequences of 8 Fuyang isolates were deposited in the GenBank database under the accession numbers: EU703812-EU703814; GQ175176-GQ175180.

\section{Phylogenetic analysis}

Alignment of the entire VP1 and 3D nucleotide sequences of the HEV71 isolates was performed using BioEdit software v5.0.9 (Tom Hall, North Carolina State University, Carolina, USA)[24]. A phylogenetic dendrogram was constructed using MEGA v4.0 program (Sudhir Kumar, Arizona State University, Arizona, USA) [25]. Similarity plot and bootscan analyses for the recombination studies were performed with SimPlot version $3.2[24,26]$. 


\section{List of abbreviations}

HFMD: Hand, foot and mouth disease; HEV71: human enterovirus 71; CV-A16: coxsackievirus A16; NNDRS: national notifiable disease report system.

\section{Competing interests}

The authors declare that they have no competing interests.

\section{Authors' contributions}

YZ, ZZ, WBX prepared manuscript. WBX and DXL designed the study and organized the coordination. $Y Z, Z Z, X J T$ performed sequence and data analysis. $Y Z$, ZZ, XJT, NYM, STX, SLZ, ALC, YZ, DMY performed RT-PCR and sequence analysis. WZY, JR, YW, OL, XPD, JZ, YPZ, JFW, ZJF, JLS, SWW collected specimens and performed virus isolation, viral identification. All authors read and approved the final manuscript.

\section{Acknowledgements}

This study was supported by a Project supported by the National Natural Science Foundation of China (Grant No. 30901259); a grant 2008BAI56B01 from the Ministry of Science and Technology of the People's Republic of China and the grants of the Key Technologies R\&D Program of National Ministry of Science 2009ZX10004-201, 2009ZX10004-202 from the People's Republic of China.

The authors thank all the provincial and prefecture laboratory staffs and epidemiologists for contributing to this HFMD outbreak investigation. The authors appreciated very much for Dr. Jane Qiu's( jane@janeqiu.com

) and Robert Fontaine's critical reviewing and good comments for this manuscript.

\section{Author Details}

'Key Laboratory for Molecular Virology \& Genetic Engineering, National Institute for Viral Disease Control and Prevention, Chinese Center for Disease Control and Prevention, Beijing 100052, China, ${ }^{2}$ Chinese Center for Disease Control and Prevention, Beijing 100050, China, ${ }^{3}$ Anhui Provincial Center for Disease Control and Prevention, Hefei 230001, China, ${ }^{4}$ Office for Disease Control and Emergency Response, Chinese Center for Disease Control and Prevention, Beijing 100050, China and 5Fuyang prefecture Center for Disease Control and Prevention, Fuyang 236000, China

Received: 8 March 2010 Accepted: 12 May 2010

Published: 12 May 2010

\section{References}

1. Schmidt RC, Maassab HF: Local immunity to influenza virus in chicken tracheal organ cultures. J Infect Dis 1974, 129:637-643.

2. Ho M, Chen ER, Hsu KH, Twu SJ, Chen KT, Tsai SF, Wang JR, Shih SR: An epidemic of enterovirus 71 infection in Taiwan. Taiwan Enterovirus Epidemic Working Group. N Engl J Med 1999, 341:929-935.

3. Chan LG, Parashar UD, Lye MS, Ong FG, Zaki SR, Alexander JP, Ho KK, Han LL, Pallansch MA, Suleiman AB, et al.: Deaths of children during an outbreak of hand, foot, and mouth disease in sarawak, malaysia: clinical and pathological characteristics of the disease. For the Outbreak Study Group. Clin Infect Dis 2000, 31:678-683.

4. McMinn P, Stratov I, Nagarajan L, Davis S: Neurological manifestations of enterovirus 71 infection in children during an outbreak of hand, foot, and mouth disease in Western Australia. Clin Infect Dis 2001, 32:236-242.

5. Wang JR, Tuan YC, Tsai HP, Yan JJ, Liu CC, Su IJ: Change of major genotype of enterovirus 71 in outbreaks of hand-foot-and-mouth disease in Taiwan between 1998 and 2000. J Clin Microbiol 2002 40:10-15

6. Chan KP, Goh KT, Chong CY, Teo ES, Lau G, Ling AE: Epidemic hand, foot and mouth disease caused by human enterovirus 71 , Singapore. Emerg Infect Dis 2003, 9:78-85.

7. Shimizu H, Utama A, Onnimala N, Li C, Li-Bi Z, Yu-Jie M, Pongsuwanna Y, Miyamura T: Molecular epidemiology of enterovirus 71 infection in the Western Pacific Region. Pediatr Int 2004, 46:231-235.

8. Zhang Y, Tan XJ, Wang HY, Yan DM, Zhu SL, Wang DY, Ji F, Wang XJ, Gao $Y J$, Chen L, et al: An outbreak of hand, foot, and mouth disease associated with subgenotype $\mathrm{C} 4$ of human enterovirus 71 in Shandong, China. J Clin Virol 2009, 44:262-267.

9. Palmenberg AC: Proteolytic processing of picornaviral polyprotein. Annu Rev Microbiol 1990, 44:603-623.

10. Li L, He Y, Yang H, Zhu J, Xu X, Dong J, Zhu Y, Jin Q: Genetic characteristics of human enterovirus 71 and coxsackievirus $A 16$ circulating from 1999 to 2004 in Shenzhen, People's Republic of China. J Clin Microbiol 2005, 43:3835-3839.

11. Yang ZH, Zhu QR, Li XZ, Wang XH, Wang JS, Hu JY, Tang W, Cui AL: [Detection of enterovirus 71 and coxsackievirus A16 from children with hand, foot and mouth disease in Shanghai, 2002]. Zhonghua Er Ke Za Zhi 2005, 43:648-652.

12. Shin SR, Ho MS, Lin KH, Wu SL, Chen YT, Wu CN, Lin TY, Chang LY, Tsao KC, Ning HC, et al:: Genetic analysis of enterovirus 71 isolated from fatal and non-fatal cases of hand, foot and mouth disease during an epidemic in Taiwan, 1998. Virus Res 2000, 68:127-136.

13. Yan JJ, Wang JR, Liu CC, Yang HB, Su IJ: An outbreak of enterovirus 71 infection in Taiwan 1998: a comprehensive pathological, virological, and molecular study on a case of fulminant encephalitis. J Clin Virol 2000, 17:13-22.

14. Ma H, He F, Wan J, Jin D, Zhu L, Liu X, Liu Q, Zhang G, Ding Z, Fontaine RE, et al.: Glucocorticoid and Pyrazolone Treatment of Acute Fever is a Risk Factor for Critical and Life-Threatening Human Enterovirus 71 Infection During an Outbreak in China, 2008. Pediatr Infect Dis J

15. Yang KD, Yang MY, Li CC, Lin SF, Chong MC, Wang CL, Chen RF, Lin TY: Altered cellular but not humoral reactions in children with complicated enterovirus 71 infections in Taiwan. J Infect Dis 2001, 183:850-856.

16. Liu ML, Lee YP, Wang YF, Lei HY, Liu CC, Wang SM, Su IJ, Wang JR, Yeh TM, Chen SH, Yu CK: Type I interferons protect mice against enterovirus 71 infection. J Gen Virol 2005, 86:3263-3269.

17. Chang LY, Hsiung CA, Lu CY, Lin TY, Huang FY, Lai YH, Chiang YP, Chiang BL, Lee CY, Huang LM: Status of cellular rather than humoral immunity is correlated with clinical outcome of enterovirus 71. Pediatr Res 2006 , 60:466-471.

18. Chan YF, AbuBaker S: Recombinant human enterovirus 71 in hand, foot and mouth disease patients. Emerg Infect Dis 2004, 10:1468-1470.

19. Yoke-Fun C, AbuBakar S: Phylogenetic evidence for inter-typic recombination in the emergence of human enterovirus 71 subgenotypes. BMC Microbiol 2006, 6:74

20. Santti J, Hyypia T, Kinnunen L, Salminen M: Evidence of recombination among enteroviruses. J Virol 1999, 73:8741-8749.

21. AbuBakar S, Wong PF, Chan YF: Emergence of dengue virus type 4 genotype IIA in Malaysia. J Gen Virol 2002, 83:2437-2442.

22. Mizuta K, Aoki Y, Suto A, Ootani K, Katsushima N, Itagaki T, Ohmi A Okamoto M, Nishimura H, Matsuzaki Y, et al.: Cross-antigenicity among EV71 strains from different genogroups isolated in Yamagata, Japan, between 1990 and 2007. Vaccine 2009, 27:3153-3158.

23. Yang CF, De L, Yang SJ, Ruiz Gomez J, Cruz JR, Holloway BP, Pallansch MA, Kew OM: Genotype-specific in vitro amplification of sequences of the wild type 3 polioviruses from Mexico and Guatemala. Virus Res 1992 24:277-296

24. Hall T: Bioedit: a user-friendly biological sequence alignment editor and analysis program forWindows 95/98/NT. Nucl Acids Symp 1999, 41:95-98.

25. Tamura KDJNM, Kumar S: MEGA4: molecular evolutionary genetics analysis (MEGA) software version 4.0. Mol Biol Evol 2007, 24:1596-1599.

26. Salminen MO, Carr JK, Burke DS, McCutchan FE: Identification of breakpoints in intergenotypic recombinants of HIV type 1 by bootscanning. AIDS Res Hum Retroviruses 1995, 11:1423-1425.

doi: 10.1186/1743-422X-7-94

Cite this article as: Zhang et al., An emerging recombinant human enterovirus 71 responsible for the 2008 outbreak of Hand Foot and Mouth Disease in Fuyang city of China Virology Journal 2010, 7:94 